\title{
RELACIÓN ENTRE LA INGESTA NUTRICIONAL Y EL RENDIMIENTO FÍSICO EN LOS ATLETAS PARTICIPANTES EN EL TRIATLÓN DEL COCO DEL AÑO 2002 EN COSTA RICA
}

\author{
Mónica Umaña Alvarado y José Moncada Jiménez \\ Escuela de Educación Física y Deportes \\ Universidad de Costa Rica, San José, Costa Rica \\ E-mail:nutridos@yahoo.com
}

\begin{abstract}
Resumen
Umaña Alvarado, M. y Moncada Jiménez, J. (2002). Relación entre la ingesta nutricional y el rendimiento físico en los atletas participantes en el Triatlón del Coco del año 2002 en Costa Rica. Revista de Ciencias del Ejercicio y la Salud, 2(2), 1-10. La investigación estudió las posibles relaciones entre el consumo de carbohidratos (CHO) antes y durante una competencia de triatlón y el rendimiento, y variación en la glicemia de los participantes. También se determinó si existían diferencias entre la ingesta de alimentos de los deportistas que presentaron síntomas gastrointestinales y los que no los presentaron. En el estudio participaron 36 triatlonistas (4 mujeres y 32 hombres), todos finalizaron la competencia. Se midió la glicemia y el peso corporal antes y después del evento. Al finalizar el evento, se aplicó una entrevista para conocer el consumo de alimentos y bebidas del desayuno, periodo de espera y durante la competencia, así como la presencia de síntomas gastrointestinales durante el evento. Se encontró que la glucosa sanguínea antes de la competencia fue menor $(M=5.49 \pm 0.65$ mmol/l) que la encontrada al finalizar la prueba $(M=6.38 \pm 1.19$ mmol/l) $(p<0.001)$. El peso al finalizar la competencia $(M=67.11 \pm 7.70 \mathrm{~kg})$ fue menor al peso inicial $(M=$ $68.53 \pm 7.74 \mathrm{~kg})(p<0.001)$. Se encontraron relaciones entre el tiempo de llegada de los participantes y el consumo de CHO $\left(\mathrm{g} \cdot \mathrm{min}^{-1}\right)$ durante el triatlón $(r=-0.42, p=0.011)$, entre la variación en la glicemia y el consumo de CHO totales $(r=0.41, p=0.013)$, y la variación en la glicemia y el consumo de $g$ CHO $\cdot \mathrm{min}^{-1}(r$ $=0.33, p=0.048$ ). No hubo casos de hipoglicemia, ni se hallaron diferencias entre la ingesta de quienes reportaron problemas gastrointestinales y los que no lo hicieron, aunque se observó una tendencia de mayor consumo de energía y nutrientes entre los que presentaron estos problemas, en comparación con los que no los presentaron. PALABRAS CLAVES: triatlón, carbohidratos, glicemia, síntomas gastrointestinales, ingesta.
\end{abstract}

\section{INTRODUCCIÓN}

En Costa Rica, el triatlón es un deporte que se ha venido practicando desde hace aproximadamente 20 años. Una de las competencias de mayor prestigio es el "Triatlón del Coco". En esta competencia, los participantes deben completar 1.6 kilómetros de natación, 60 kilómetros de ciclismo y 16 kilómetros de atletismo. La prueba se desarrolla en playas del Coco, en la provincia de Guanacaste, a partir de las doce mediodía y esto ocasiona que los atleta compitan bajo un alto estrés ambiental. Según el ACSM (1996), el índice de temperatura del globo de bulbo húmedo (TGBH) es el índice ambiental más utilizado para determinar el estrés por calor en las competencias. Cuando el TGBH sobrepasa los $28{ }^{\circ} \mathrm{C}$, el riesgo de cansancio o agotamiento por calor y golpe de calor es muy alto.

En el Triatlón del Coco, los participantes duran entre tres y cinco horas en finalizar el evento. Cuando se compite en eventos deportivos a intensidades moderadas y altas $\left(70 \%-90 \% \mathrm{VO}_{2 \text { máx }}\right)$, en las que la duración del esfuerzo físico es igual o mayor de dos horas, el consumo adecuado de carbohidratos $(\mathrm{CHO})$ antes y/o durante el evento ejerce efectos positivos en el rendimiento deportivo. Según Hargreaves (1997), el objetivo de la suplementación con $\mathrm{CHO}$ durante el ejercicio prolongado es el de proveer al organismo de glucosa suficiente para mantener su concentración sanguínea en un rango normal, el cual, de acuerdo con el 
American College of Sports Medicine (ACSM) (2000), varía entre 3.8 y 6.04 $\mathrm{mmol} / \mathrm{l}$. Además, la ingesta de $\mathrm{CHO}$ durante el ejercicio promueve la oxidación de la glucosa y otros $\mathrm{CHO}$ exógenos sin ocasionar problemas gastrointestinales ni transporte inadecuado de fluidos. Para lograr estos objetivos, se ha recomendado una ingesta de 1.0 a $1.5 \mathrm{~g} \mathrm{CHO} \cdot \mathrm{min}^{-1}$ durante el ejercicio prolongado (Jeukendrup y Jentjens, 2000).

Una ingesta reducida de $\mathrm{CHO}$ conlleva a una menor disponibilidad de glucosa sanguínea para ser oxidada, situación que puede acarrear problemas médicos como la hipoglicemia (glucosa sanguínea $<3.8$ $\mathrm{mmol} / \mathrm{l}$ ). Por el contrario, una ingesta elevada de CHO disminuye la eficiencia en la oxidación de $\mathrm{CHO}$ en relación con la cantidad consumida (Jeukendrup, Vet-Joop, Sturk, Stegen, Senden, Saris, y Wagenmakers, 2000), y puede ocasionar problemas gastrointestinales que afecten el desarrollo de la prueba o impidan su finalización.

La última comida precompetencia influye en el rendimiento deportivo, en especial la composición nutricional y hora de consumo de la misma. Con el fin de mejorar el rendimiento se recomienda ingerir aproximadamente $200-300 \mathrm{~g}$ de $\mathrm{CHO}$ durante las 4 horas previas al ejercicio, ya que optimiza el suministro de glucógeno muscular y glucosa sanguínea en las últimas etapas del ejercicio (Coyle, 1995). De acuerdo con el ACSM, la American Dietetic Association (ADA) y Dietitians of Canada (DOC) (2000), la comida precompetencia debe poseer las siguientes características. Primero, debe suministrar fluidos, los cuales son importantes para el atleta porque le permiten comenzar la competencia en un estado de euhidratación. Segundo, la comida precompetencia debe estar compuesta de una cantidad baja en grasas y fibra para facilitar el vaciamiento gástrico y minimizar problemas gastrointestinales. Tercero, debe poseer un alto contenido de $\mathrm{CHO}$ con el propósito de mantener normales los niveles de glucosa sanguínea y maximizar las reservas de glucógeno. Finalmente, la comida precompetencia debe ser moderada en proteína y compuesta de alimentos familiares para el atleta.

En eventos de resistencia como el triatlón, entre el $30 \%$ y el $50 \%$ de los participantes pueden sufrir uno o más síntomas gastrointestinales, como resultado de maladigestión, malabsorción, cambios en el tránsito del intestino delgado e ingesta inapropiada de alimentos y líquidos (Brouns y Beckers, 1993).

El ejercicio prolongado promueve una redistribución del flujo sanguíneo, aumentando el flujo a los músculos activos y a la piel. Como consecuencia se reduce el aporte sanguíneo a otros órganos como hígado, riñones, tejido inactivo e intestino. En el intestino, el flujo sanguíneo disminuido puede afectar la absorción de CHO y otros nutrientes, ocasionando una reducción en la tasa de oxidación de $\mathrm{CHO}$ exógenos (Gisolfi, 2000).

Cuando el ejercicio en el calor está acompañado de deshidratación (pérdida $>3.0$ $\%$ de la masa corporal), las funciones circulatorias y termorregulatorias se deterioran aún más y el intestino puede estar sujeto a los efectos combinados de isquemia, hipoxia e hipertermia. Bajo estas condiciones, la función de la barrera intestinal puede estar comprometida, llevando a necrosis tisular, endotoxemia y deterioro circulatorio (Gisolfi, 2000).

La presente investigación pretendió en primera instancia, examinar si existían diferencias estadísticamente significativas entre la ingesta alimentaria de los atletas que presentaban hipoglicemia o síntomas gastrointestinales y los que no presentaron dichos problemas médicos. Segundo, se estudió la relación entre el rendimiento físico en una competencia de campo (i.e., Triatlón del Coco) y la ingesta de $\mathrm{CHO}$ y otros nutrientes antes y durante la misma. Finalmente, se quiso determinar si existía alguna relación entre la variación en las concentraciones de glucosa sanguínea de los atletas desde el inicio hasta el final del evento y el consumo de $\mathrm{CHO}$ y otros nutrientes antes y durante el triatlón. 


\section{METODOLOGÍA}

\section{Sujetos}

Treinta y seis triatlonistas, 4 mujeres y 32 hombres, participaron voluntariamente en el estudio. Las características de los sujetos se presentan en la Tabla 1. Antes de iniciar el triatlón, cada atleta recibió una explicación del propósito del estudio y firmó un consentimiento informado. Para participar como sujeto de investigación, era indispensable que el atleta finalizara el evento.

\section{Instrumentos de medición}

Se midió el índice de temperatura del globo de bulbo húmedo (TGBH) utilizando un monitor WiBGeT Mod-RSS-214. Según el ACSM (1996), el TGBH es el índice ambiental más utilizado para determinar el estrés por calor en las competencias.

La concentración de glucosa sanguínea fue medida utilizando un glucómetro marca Bayer ${ }^{\circledR}$, modelo Advantage. El glucómetro presentaba los valores en $\mathrm{mg} / \mathrm{dl}$; sin embargo, éstos fueron transformados a unidades internacionales $(\mathrm{mmol} / \mathrm{l})$ para su posterior análisis. El peso corporal $(\mathrm{kg})$ se midió en una balanza electrónica marca A\&D, modelo UC-300 (Engineering, Inc., USA).

La información acerca del consumo de alimentos fue recopilada por medio de una entrevista, empleando un cuestionario. Para la estimación de cantidades de alimentos se utilizaron dos catálogos: el Manual de Fotografías de Porciones de Alimentos y Preparaciones Comunes en Costa Rica y Equivalencias de Listas de Intercambio (Chinnock y Sedó, 2001), y un catálogo de productos que incluía las etiquetas de barras energéticas y geles disponibles en Costa Rica. Este último se diseñó en forma exclusiva para la investigación.

Los síntomas gastrointestinales fueron registrados por medio de un cuestionario de identificación de problemas gastrointestinales, que además incluía el registro del peso corporal y las concentraciones de glucosa sanguínea. El reporte oficial del evento con los tiempos de llegada de los participantes fue suministrado por los organizadores de la competencia.

\section{Procedimientos}

Una hora antes de iniciar la competencia, los atletas colocaban sus bicicletas en un espacio destinado para tal fin, el cual estaba localizado a $100 \mathrm{~m}$ del lugar donde se realizaría la competencia de natación. Cuando los sujetos iban a ubicar sus bicicletas en el sitio correspondiente, los investigadores obtuvieron los valores de la glicemia y el peso corporal de cada participante.

Para obtener los valores de la glicemia de los sujetos, se les limpiaba el dedo índice de la mano derecha con algodón y alcohol. Luego, con una lanceta esterilizada se punzaba el dedo y se obtenía una gota de sangre. Esta se recogía en una cinta reactiva y se insertaba en el analizador. Al cabo de unos segundos, el analizador mostraba el resultado en una pantalla de cristal líquido. Para obtener el peso corporal, se le pedía a los sujetos quitarse la mayor cantidad de ropa posible. En la mayoría de los casos, los sujetos fueron pesados vistiendo únicamente el traje de baño.

Los procedimientos anteriores se repitieron inmediatamente después de terminar la competencia. Además, conforme los sujetos terminaron el evento, fueron entrevistados para registrar la ingesta de alimentos y bebidas del desayuno, el periodo de espera y durante la competencia, en los trayectos de ciclismo y atletismo.

Para estimar el volumen de bebidas hidratantes consumido, se contó con varios tamaños de ánforas y se registraron las cantidades en polvo empleadas para preparar las bebidas. En la última parte de la entrevista se registraron los síntomas gastrointestinales presentes en alguna de las tres disciplinas.

\section{Análisis estadístico}

Se utilizó el programa de análisis dietético en Epi-Info (Ulate y Chinnock, 
2002) para efectuar el análisis nutricional. También se empleó el programa estadístico SPSS $^{\circledR}$ versión 8.0 para calcular los promedios $(M)$ y desviaciones estándar $( \pm$ $D S$ ) de las características de los participantes, así como de la energía, macronutrientes consumidos, y síntomas gastrointestinales reportados. Se calcularon correlaciones producto momento de Pearson entre las concentraciones de glucosa sanguínea y el rendimiento, y entre el consumo de $\mathrm{CHO}$ y el rendimiento. Se utilizó la prueba $t$ student para mediciones repetidas con el fin de determinar si existían diferencias significativas entre la medición inicial y final de la concentración de glucosa sanguínea y del peso corporal. Se empleó la prueba $t$ student para grupos independientes para analizar diferencias significativas entre el consumo de energía y nutrientes de los grupos con o sin presencia de síntomas gastrointestinales.

\section{RESULTADOS}

Treinta y seis sujetos participaron en el estudio, de los cuales 10 pertenecían a la categoría élite y 26 a categorías por edad. La muestra representa un $44 \%$ del total de triatlonistas que participaron en el evento.

El TGBH alcanzó los $30.1^{\circ} \mathrm{C}$ al inicio de la competencia a las 12:30 p.m. El TGBH promedio durante la competencia fue de $31.8^{\circ} \mathrm{C}$.

Por medio de la prueba $t$ student de medidas repetidas se encontró una diferencia estadísticamente significativa en las concentraciones de glucosa sanguínea $(p<$ 0.001). El promedio de la glucosa sanguínea antes de la competencia fue $M=5.49 \pm 0.65$ $\mathrm{mmol} / \mathrm{l}$, mientras después de la competencia fue $M=6.38 \pm 1.19 \mathrm{mmol} / \mathrm{l}$. En promedio, la glicemia aumentó $M=0.89 \pm 1.29 \mathrm{mmol} / \mathrm{l}$. Ningún sujeto estudiado presentó hipoglicemia al finalizar el evento ya que el valor mínimo encontrado fue $4.27 \mathrm{mmol} / \mathrm{l}$.

Al analizar el peso corporal de los sujetos por medio de la prueba $t$ student de medidas repetidas, se encontró que el peso corporal al finalizar la competencia $(M=$ $67.11 \pm 7.70 \mathrm{~kg}$ ) fue estadísticamente menor al peso inicial $(M=68.53 \pm 7.74 \mathrm{~kg})(p<$ $0.001)$. Cabe anotar que esta reducción de $1.44 \%$ de la masa corporal pudo estar influenciada por la medición final del peso corporal. En este estudio de campo, los sujetos fueron pesados al final de la competencia con sus respectivos trajes de baño, los cuales acumulaban líquidos (e.g., agua, sudor), por lo que los valores podrían subestimar la verdadera pérdida de peso.

En la Tabla 2 se reportan los problemas gastrointestinales más comunes y el número de sujetos que presentaron problemas en alguna de las disciplinas del triatlón. Veintisiete sujetos $(75 \%)$ reportaron problemas gastrointestinales; siendo durante el segmento de ciclismo y atletismo cuando se presentaron más frecuentemente los síntomas de eructación, cólico e inflamación estomacal. Los sujetos presentaron la mayor cantidad de problemas durante el atletismo (63.89\%), en comparación con el ciclismo $(47.2 \%)$ y la natación $(21.6 \%)$.

Tabla 1. Características de los participantes en el Triatlón del Coco 2002. $(n=36)$

\begin{tabular}{|c|c|c|c|}
\hline Variable & Promedio \pm DS & Mínimo & Máximo \\
\hline Edad (años) & $30.61 \pm 9.87$ & 19 & 64 \\
\hline Número de años practicando triatlón & $6.38 \pm 4.97$ & 1 & 20 \\
\hline Número de triatlones del Coco finalizados & $3.17 \pm 3.62$ & 0 & 14 \\
\hline Entrenamiento natación $\left(\mathrm{h} \cdot \mathrm{sem}^{-1}\right)$ & $4.07 \pm 1.77$ & 1 & 9 \\
\hline Entrenamiento ciclismo $\left(\mathrm{h} \cdot \mathrm{sem}^{-1}\right)$ & $7.15 \pm 3.62$ & 2 & 20 \\
\hline Entrenamiento atletismo $\left(\mathrm{h} \cdot \mathrm{sem}^{-1}\right)$ & $3.69 \pm 1.58$ & 1 & 8 \\
\hline Tiempo de arribo a la meta (min) & $237 \pm 33$ & 183 & 312 \\
\hline
\end{tabular}


Tabla 2. Frecuencia (f) de problemas gastrointestinales (GI) reportados por los triatlonistas durante el Triatlón del Coco 2002.

\begin{tabular}{|c|c|c|c|c|c|c|}
\hline \multirow{2}{*}{ Síntoma gastrointestinal } & \multicolumn{2}{|c|}{ Natación } & \multicolumn{2}{|c|}{ Ciclismo } & \multicolumn{2}{|c|}{ Atletismo } \\
\hline & $\mathbf{f}$ & $\%$ & $\mathbf{f}$ & $\%$ & f & $\%$ \\
\hline Eructación & 1 & 2.70 & 8 & 21.6 & 8 & 21.60 \\
\hline Dolor abdominal/cólico & 4 & 10.81 & 3 & 8.11 & 9 & 24.32 \\
\hline Inflamación estomacal & 1 & 2.70 & 3 & 8.11 & 6 & 16.22 \\
\hline Urgencia para defecar & 0 & 0.00 & 1 & 2.70 & 5 & 13.51 \\
\hline Calambres estomacales/intestinales & 1 & 2.70 & 1 & 2.70 & 4 & 10.81 \\
\hline Náuseas & 1 & 2.70 & 0 & 0.00 & 2 & 5.41 \\
\hline Vómito & 1 & 2.70 & 0 & 0.00 & 2 & 5.41 \\
\hline Urgencia para vomitar & 1 & 2.70 & 1 & 2.70 & 0 & 0.00 \\
\hline Flatulencia & 0 & 0.00 & 1 & 2.70 & 1 & 2.70 \\
\hline Diarrea & 0 & 0.00 & 0 & 0.00 & 1 & 2.70 \\
\hline Total de síntomas GI reportados = & 10 & & 18 & & 38 & \\
\hline Total de sujetos con síntomas GI = & 8 & 21.60 & 17 & 47.20 & 23 & 63.89 \\
\hline
\end{tabular}

El tiempo promedio transcurrido entre el desayuno de los atletas y el inicio de la competencia fue $M=244 \pm 74 \mathrm{~min}$. En la Tabla 3 se presenta el análisis nutricional del periodo precompetencia (i.e., desayuno y periodo de espera), y durante la competencia, en las secciones de ciclismo y atletismo. Se compara la ingesta de energía, $\mathrm{CHO}$, grasa, proteína y fibra entre los atletas que presentaron y los que no presentaron problemas gastrointestinales durante la competencia.

No se encontraron diferencias estadísticamente significativas en la ingesta de los dos grupos; sin embargo, se encontró una tendencia que sugiere que los atletas con síntomas gastrointestinales presentaban mayores consumos de fibra durante el ciclismo $(p=0.066)$ y durante el atletismo ( $p$ $=0.054)$.

Respecto a la ingesta de $\mathrm{CHO}$ durante la competencia en función del tiempo, se encontró que 12 sujetos (33.33\%) ingirieron $<0.5 \mathrm{~g} \cdot \mathrm{min}^{-1}, 17$ sujetos $(47.22 \%)$, entre 0.5 - $1.0 \mathrm{~g} \cdot \min ^{-1}$ y 7 sujetos $(19.44 \%)$, indicaron ingestas $>1.0 \mathrm{~g} \cdot \mathrm{min}^{-1}$.

Por medio de la correlación de Pearson, se encontró una relación inversa significativa entre el tiempo de competencia y la cantidad de $\mathrm{CHO}$ consumidos $\left(\mathrm{g} \cdot \mathrm{min}^{-1}\right)$ durante el evento $(r=-0.42, p=0.011)$ (Figura 1). No se encontró una relación entre el consumo de $\mathrm{CHO}$ precompetencia y el rendimiento.

Tabla 3. Análisis nutricional $(M \pm D S)$ de la ingesta de triatlonistas, antes y durante el Triatlón del Coco 2002, según presencia de síntomas gastrointestinales.

\begin{tabular}{cccccc}
\hline Evento & $\begin{array}{c}\text { Energía } \\
(\mathbf{k J})\end{array}$ & $\begin{array}{c}\text { CHO } \\
(\mathbf{g r})\end{array}$ & $\begin{array}{c}\text { Grasa } \\
(\mathbf{g r})\end{array}$ & $\begin{array}{c}\text { Proteína } \\
(\mathbf{g r})\end{array}$ & $\begin{array}{c}\text { Fibra } \\
(\mathbf{g r})\end{array}$ \\
\hline Precompetencia & & & & & \\
Con síntomas (n=27) & $4632 \pm 1948$ & $208.40 \pm 142.90$ & $21.86 \pm 13.49$ & $25.02 \pm 13.74$ & $7.20 \pm 4.26$ \\
Sin síntomas (n=9) & $4281 \pm 1488$ & $188.47 \pm 72.21$ & $18.18 \pm 10.42$ & $23.93 \pm 12.42$ & $6.36 \pm 2.40$ \\
$\quad \begin{array}{l}\text { Ciclismo } \\
\text { Con síntomas (n=27) }\end{array}$ & $2120 \pm 1146$ & $115.79 \pm 61.25$ & $1.68 \pm 2.29$ & $3.35 \pm 6.22$ & $0.72 \pm 1.08$ \\
Sin síntomas (n=9) & $1493 \pm 1142$ & $81.32 \pm 67.27$ & $2.17 \pm 3.31$ & $2.70 \pm 4.24$ & $0.22 \pm 0.47$ \\
$\quad \begin{array}{ll}\text { Atletismo } \\
\text { Con síntomas (n=27) }\end{array}$ & $882 \pm 707$ & $49.76 \pm 37.94$ & $0.27 \pm 0.94$ & $1.70 \pm 3.27$ & $0.60 \pm 1.32$ \\
Sin síntomas (n=9) & $866 \pm 832$ & $48.18 \pm 43.85$ & $0.009 \pm 0.27$ & $1.22 \pm 2.31$ & $0.008 \pm 0.16$ \\
\hline Nis CHO
\end{tabular}

Nota: $\mathrm{CHO}=$ carbohidratos 
Figura 1. Diagrama de dispersión que muestra la relación entre el tiempo de competencia y el consumo de carbohidratos de los participantes en el Triatlón del Coco 2002.

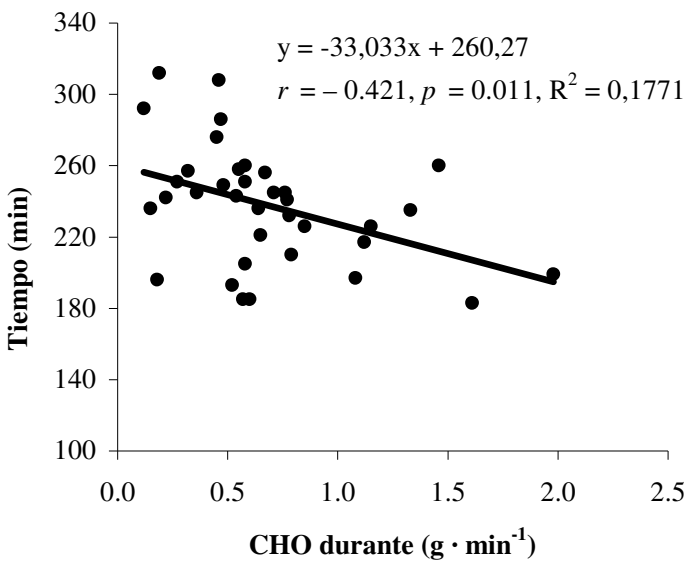

Se encontró una relación significativa entre la variación de la glucosa sanguínea y el consumo de $\mathrm{CHO}$ durante la prueba, tanto para la ingesta total en gramos $(\mathrm{r}=0.41, \mathrm{p}=$ $0.013)$, como para la ingesta en $\mathrm{g} \cdot \mathrm{min}^{-1}(\mathrm{r}=$ $0.33, p=0.048)$. La Figura 2 muestra la relación entre el cambio en la glicemia y el consumo total de $\mathrm{CHO}$ durante la competencia, mientras que la Figura 3 presenta la relación entre el cambio en la glicemia y el consumo de $\mathrm{g} \mathrm{CHO} \cdot \mathrm{min}^{-1}$.

Figura 2. Diagrama de dispersión entre la variación de la glucosa sanguínea y consumo total de CHO de los participantes en el Triatlón del Coco 2002.

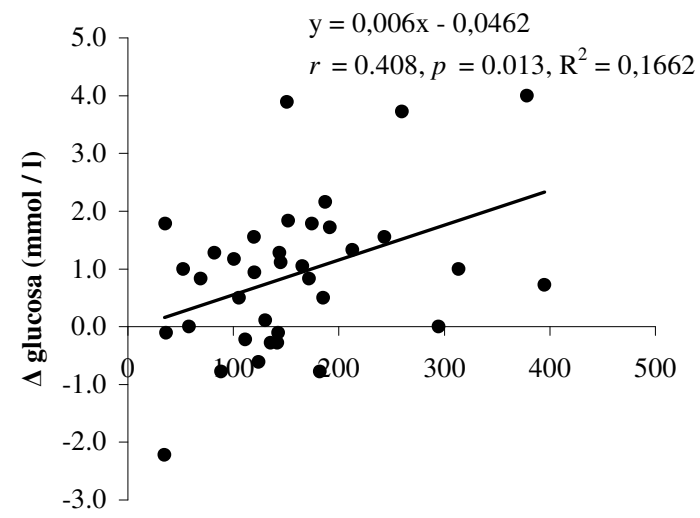

CHO durante (gr)
Figura 3. Diagrama de dispersión entre la variación de la glucosa sanguínea y consumo de CHO $\cdot \min ^{-1}$ de los participantes en el Triatlón del Coco 2002.

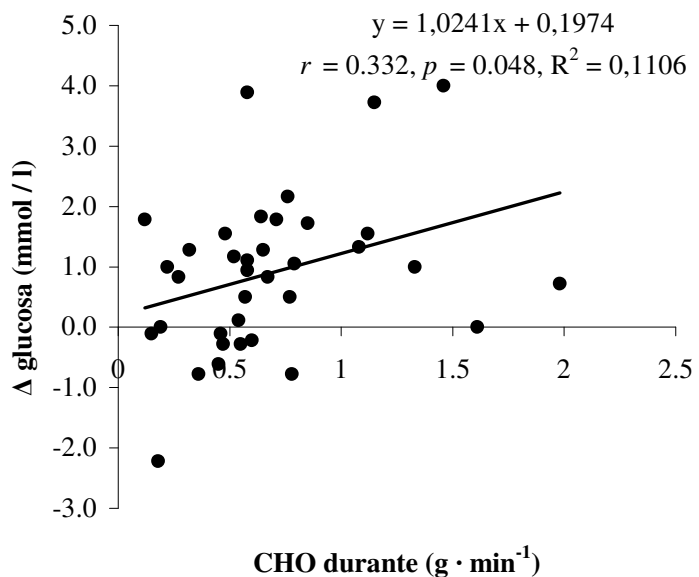

\section{DISCUSIÓN}

Una de las características del Triatlón del Coco es el estrés ambiental en el que compiten los atletas. Durante este estudio, los sujetos compitieron a un alto estrés ambiental, ya que el TGBH alcanzó los 30.1 ${ }^{\circ} \mathrm{C}$ al inicio de la competencia. Existe un número considerable de países latinoamericanos ubicados en la región tropical y no es extraño encontrar valores del TGBH mayores a $28{ }^{\circ} \mathrm{C}$, especialmente al nivel del mar (Aragón et al., 1999). Se recomienda a los países del trópico seguir las pautas del ACSM. Costa Rica, por ser un país tropical, debería adoptar dichas pautas, en las cuales se recomienda posponer la carrera hasta que prevalezcan condiciones menos estresantes, se vuelva a programar, o se cancele. En caso de llevar a cabo el evento, los participantes deben estar enterados del riesgo y tomar medidas para minimizar el impacto del estrés por calor.

La combinación de estrés ambiental y competencias en las que se debe recorrer largas distancias exige que los atletas apliquen estrategias nutricionales para terminar el evento en condiciones aceptables. La fatiga que ocurre en ejercicios prolongados parece ser el resultado de dos causas principales, la depleción de las 
reservas de glucógeno y la deshidratación (Villa, 2000). Por ello, las estrategias nutricionales buscan prevenir las causas de fatiga e incluyen un protocolo de hidratación, alimentación precompetencia adecuada y consumo recomendado de $\mathrm{CHO}$ durante la prueba.

Los CHO son el mayor combustible utilizado durante entrenamiento y competencia en ejercicio prolongado (Sherman y Lamb, 1988). Ya que los CHO representan una fuente energética tan importante en ejercicio de larga duración, su consumo es fundamental porque ayuda a mantener la concentración de glucosa sanguínea, así como una tasa constante de oxidación de éstos.

Varios autores (Sherman y Lamb, 1988; Coggan y Swanson, 1992; Coyle, 1995; Hargreaves, 2001; Jeukendrup y Jentjens, 2000; Aragón, 2001), afirman que el consumo de $\mathrm{CHO}$ durante el ejercicio prolongado de intensidad moderada a alta mejora el rendimiento físico. Coggan y Swanson (1992), señalan que dicha mejora puede deberse a la habilidad del organismo para mantener una intensidad dada de ejercicio durante más tiempo, así como a la capacidad para ejercitarse a intensidades mayores en las últimas etapas del ejercicio prolongado.

En el presente estudio se estimó el consumo de $\mathrm{CHO}$ de los triatletas antes y durante la competencia. El consumo promedio precompetencia fue de 203 gramos, el cual se llevó a cabo en el transcurso de las 4 horas anteriores a la competencia, y se ubica dentro del rango de $200-300 \mathrm{~g}$ recomendado por Coyle (1995). Sin embargo, en el presente estudio esta recomendación no se relacionó con el rendimiento.

La suplementación recomendada de $\mathrm{CHO}$ durante el ejercicio se expresa en masa (i.e, g) por unidad de tiempo (i.e., $\min ^{-1} \mathrm{u} \mathrm{h}^{-1}$ ). El consumo promedio de $\mathrm{CHO}$ de los triatlonistas durante la competencia fue de $0.68 \mathrm{~g} \cdot \mathrm{min}^{-1}$. Se ha encontrado que la ingesta de 1.0 a $1.5 \mathrm{~g} \cdot \min ^{-1}$ aumenta la tasa de oxidación muscular de 1.0 a $1.1 \mathrm{~g} \cdot \mathrm{min}^{-1}$. Una ingesta mayor de $\mathrm{CHO}$ no eleva las tasas de oxidación (Jeunkendrup y Jentjens, 2000).
Del grupo de deportistas en estudio, solamente $7(21 \%)$ consumieron más de 1.0 $\mathrm{g} \cdot \mathrm{min}^{-1}$, cantidad mínima recomendada para optimizar la oxidación muscular de $\mathrm{CHO}$ exógenos. Esto implica que los triatlonistas podrían utilizar al máximo los $\mathrm{CHO}$ como fuente de energía si cumplieran con esta recomendación. De esta manera es posible mantener la intensidad deseada del ejercicio y retardar la aparición de la fatiga.

Se analizaron posibles relaciones entre consumo de $\mathrm{CHO}$ y el rendimiento o variación de la glucosa sanguínea. Se encontró una relación entre el consumo $\mathrm{CHO}$ durante la competencia $\left(\mathrm{g} \cdot \mathrm{min}^{-1}\right)$ y el rendimiento en el triatlón. En la Figura 1 se observa una relación inversa, moderada y significativa, en donde a mayor consumo de carbohidratos también se observa una menor duración para completar el triatlón. Según Aragón (2001), la contribución de los CHO como combustible durante el ejercicio depende de la intensidad del ejercicio y de la condición física de los sujetos, entre otros. Es importante considerar que en este estudio se asumió que los sujetos compitieron a intensidades fuertes $\left(70 \%-90 \% \mathrm{VO}_{2 \text { máx }}\right)$, no se determinó la intensidad promedio a la que se ejercitaron, por lo que se sugiere tomar en cuenta esta variable para futuras investigaciones. El estado de entrenamiento también influye en la utilización de CHO, ya que el entrenamiento de resistencia produce adaptaciones a nivel muscular, permitiendo ahorrar glucógeno (Villa, 2000).

El consumo de $\mathrm{CHO}$ durante la competencia influyó positivamente en la concentración de glucosa sanguínea, tal y como se muestra en las Figuras 2 y 3 . En eventos prolongados, los niveles de glucosa sanguínea se mantienen gracias a la liberación endógena de la glucosa hepática, vía glucogenólisis y gluconeogénesis, y a la ingestión de glucosa exógena (Fallon, Broad, Thompson y Reull, 1998). Sin embargo, las reservas de glucógeno hepático son limitadas, por lo que el consumo de $\mathrm{CHO}$ contribuye a mantener los niveles de glucosa sanguínea. Widrick, Costill, Fink, Hickey, McConell y Tanaka (1993), investigaron los efectos de la concentración inicial de glucógeno muscular 
sobre el rendimiento, y hallaron un efecto ergogénico de la suplementación con $\mathrm{CHO}$ cuando las reservas previas de glucógeno muscular eran bajas. Asimismo, midieron los niveles de glucosa sanguínea y encontraron que ésta disminuyó cuando no hubo suplementación de $\mathrm{CHO}$, sin importar si las reservas previas de glucógeno muscular eran altas o bajas. Este hallazgo claramente indica que el consumo de $\mathrm{CHO}$ juega un papel más determinante en el mantenimiento de la glucosa sanguínea, que los niveles previos de glucógeno muscular, para ejercicios realizados durante al menos 2 horas a un 70 $\%$ del $\mathrm{VO}_{2 \text { máx }}$.

En la presente investigación, ningún sujeto estudiado presentó hipoglicemia. Nueve atletas $(27 \%)$ presentaron una disminución en la glucosa sanguínea al finalizar el evento. Sin embargo, la glucosa sanguínea aumentó en promedio $0.89 \mathrm{mmol} / \mathrm{l}$. Este aumento puede deberse a varias razones. Primero, al consumo de $\mathrm{CHO}$ antes y durante la competencia. Segundo, a las reservas previas de glucógeno muscular, las cuales no fueron medidas en el estudio. Tercero, al incremento en la concentración de hormonas del estrés. Según Davis y Brown (2002), el sistema endocrino trata de mantener niveles normales de glucosa sanguínea durante el ejercicio, movilizando otras fuentes de energía y estimulando la producción de glucosa a partir de aminoácidos y otras fuentes. Cuarto, al aumento en la concentración de solutos en el volumen sanguíneo (hemoconcentración) como consecuencia de la deshidratación, lo que hace que haya mayor cantidad de glucosa por litro de sangre.

El $75 \%$ de los sujetos reportaron problemas gastrointestinales, demostrando lo común que fue el problema en este grupo de deportistas. Este dato concuerda con valores señalados en otras investigaciones. Por ejemplo, Jeukendrup et al. (2000), encontraron que el $93 \%$ de los participantes de un triatlón Ironman ${ }^{\circledR}$ presentaron síntomas gastrointestinales, mientras que Worme, Doubt, Singh, Ryan, Moses y Deuster (1990), hallaron que el $50 \%$ de los triatlonistas entrevistados $(n=33)$ reportaron síntomas gastrointestinales en entrenamientos y competencias.

Una de las causas de los problemas gastrointestinales reportados en pruebas de larga duración es el consumo excesivo de $\mathrm{CHO}$ antes y durante el ejercicio. El consumo de altas concentraciones de azúcar puede causar un efecto osmótico y retener un exceso de fluidos en el estómago o intestinos, lo que podría causar problemas gastrointestinales y disminución en el rendimiento (Williams, 1995). Sin embargo, no se encontró una diferencia estadísticamente significativa en el consumo de $\mathrm{CHO}$ entre los atletas que presentaron y los que no presentaron problemas gastrointestinales, que pudiera explicar la presencia de síntomas.

Jeukendrup y Jentjens (2000), afirman que los $\mathrm{CHO}$ pueden ser ingeridos a tasas altas de hasta $3.0 \mathrm{~g} \cdot \mathrm{min}^{-1}$ antes de causar síntomas gastrointestinales. Según lo anterior, el consumo de $\mathrm{CHO}$ durante la competencia no hubiera sido la causa de los síntomas ya que la cantidad mayor de $\mathrm{CHO}$ ingeridos fue de $1.98 \mathrm{~g} \cdot \mathrm{min}^{-1}$.

Por otra parte, el consumo de fibra durante la competencia puede ocasionar trastornos gastrointestinales al retrasar el vaciamiento gástrico y causar una sensación de llenura que dificulte la alimentación e hidratación. En esta investigación, las diferencias en el consumo promedio de fibra en el ciclismo y el atletismo entre los dos grupos, estuvieron muy cerca de ser estadísticamente significativas, lo que sugiere una tendencia importante entre el grupo que presentó y el que no presentó problemas gastrointestinales. La diferencia promedio en el consumo de fibra en el ciclismo fue $0.50 \mathrm{~g}$, mientras que la diferencia promedio en el atletismo fue $0.59 \mathrm{~g}$. A pesar de que las diferencias en la ingesta de fibra entre los dos grupos fue estadísticamente significativa, las cantidades son muy pequeñas para ocasionar trastornos gastrointestinales, por lo que el recomendar abstenerse o consumir la menor cantidad posible de alimentos fuente de fibra dietética durante la carrera es debatible en este caso. Los alimentos que aportaron fibra en el presente estudio fueron las frutas (e.g., 
banano, papaya, sandía, y manzana) y las barras (energéticas y de granola).

En el Cuadro 3, al comparar la ingesta de energía y nutrientes según la presencia de síntomas gastrointestinales, se observa que el consumo promedio de energía $\mathrm{y}$ nutrientes fue mayor en el grupo que presentó malestares, salvo el consumo de grasa durante la etapa de ciclismo, siendo mayor en el grupo que no presentó problemas.

Con base en la información anterior, es claro indicar que debe considerarse la existencia de otras causas de la aparición de problemas gastrointestinales, como es el caso del estrés competitivo y la endotoxemia, variables que no fueron medidas en esta investigación pero que merecen mayor atención.

En conclusión, el rendimiento en la competencia mejoró a medida que aumentaba el consumo de $\mathrm{CHO}\left(\mathrm{g} \cdot \mathrm{min}^{-1}\right)$, mientras que la variación en la glicemia se relacionó positivamente con el consumo de $\mathrm{CHO}$ durante la prueba, tanto en el consumo total como en $\mathrm{g} \cdot \mathrm{min}^{-1}$. El consumo promedio de CHO resultó ser menor que las recomendaciones para optimizar la oxidación de carbohidratos. No se encontró diferencia entre el consumo de los atletas que reportaron síntomas gastrointestinales y los que no los presentaron. No obstante, la fibra estuvo cerca de mostrar una diferencia significativa, por lo que se requiere mayor investigación en esta área, especialmente en cuanto al tipo de fibra consumida durante la competencia. También se requiere más investigación con respecto a las recomendaciones de consumo de proteínas, grasa y fibra antes y durante el ejercicio, ya que a diferencia de los carbohidratos, no existen cantidades establecidas de esos nutrientes.

Agradecimiento. A la Federación Costarricense de Triatlón, a la Sra. Ana Teresa Garita, Prof. Anne Chinnock, Sra. Lilliana Pérez, al Sr. Salvador Alvarado, Sr. Gerardo Serrato, Sr. Carlos Sánchez, y al equipo que colaboró en la recolección de datos.

\section{REFERENCIAS}

American College of Sports Medicine, American Dietetic Association \& Dietitians of Canada. (1996). Position Stand: Heat and cold illnesses during distance running. Medicine and Science in Sports and Exercise, 28(12), i-x.

American College of Sports Medicine. (2000). ACSM's guidelines for exercise testing and prescription $\left(6^{\text {th }}\right.$ ed.). Baltimore, MD: Williams \& Wilkins.

American College of Sports Medicine, American Dietetic Association \& Dietitians of Canada. (2000). Nutrition and Athletic Performance. Medicine and Science in Sports and Exercise, 32(12), 2130-2145.

Aragón, L., Maughan, R., Rivera-Brown, A., Meyer, F., Murray, R., de Barros, T., García, P., Sarmiento, J., Arroyo, F., Javornik, R., Matsudo, V., Salazar, W. \& Lentini, N. (1999). Actividad física en el calor: termorregulación e hidratación en América Latina. En: Gatorade Sports Science Institute (Ed.), Bases de nutrición deportiva para el inicio del nuevo milenio (pp. 9-19). Argentina: Biosystem Servicio Educativo.

Aragón, L. (2001). Metabolic and performance responses to carbohydrate intake during exercise. En Maughan, R \& Murray, R. (Eds.), Sports drinks: basic science and practical aspects (pp.153-180). Boca Ratón, FL: CRC Press.

Brouns, F. \& Beckers, E. (1993). Is the gut an athletic organ? Sports Medicine, 15(4), 242-257.

Chinnock, A. \& Sedó, P. (2001). Manual de Fotografías de Porciones de Alimentos y Preparaciones Comunes en Costa Rica y Equivalencias de Listas de Intercambio. San José, Costa Rica: Universidad de Costa Rica.

Coggan, A. \& Swanson, S. (1992). Nutritional manipulations before and during endurance exercise: effects on performance. Medicine and Science in Sports and Exercise, 24(9), s331-s335.

Coyle, E. (1995). Substrate utilization during exercise in active people. American Journal of Clinical Nutrition, 61(suppl), 968s-979s.

Davis, M. \& Brown, A. (2002). Carbohydrates, hormones and endurance performance. Sports Science Exchange \#80,14(1). Disponible en: http://www.gssiweb.com

Fallon, K., Broad, E., Thompson, M. \& Reull, P. (1998). Nutritional and fluid intake in a $100-\mathrm{km}$ ultramarathon. International Journal of Sport Nutrition, 8, 24-35.

Gisolfi, C. (2000). Is the GI system built for exercise? News of Physiology Science, 15, 114-119.

Hargreaves, M. (1997). Metabolic responses to carbohydrate ingestion: effects on exercise performance. En Lamb, D. \& Murray, R (Eds.), Perspectives in Exercise Science and Sports Medicine: Optimizing sport performance (pp.93119). Dallas, TX: Cooper Publishing Group.

Hargreaves, M. (2001). Carbohydrate ingestion and exercise: effects on metabolism and performance. 
Sports Science Exchange \#75,12(4). Disponible en: http://www.gssiweb.com

Jeukendrup, A. \& Jentjens, R. (2000). Oxidation of carbohydrate feedings during prolonged exercise. Sports Medicine, 29(6), 407-424.

Jeukendrup, A., Vet-Joop, K., Sturk, A., Stegen, J., Senden, J., Saris, W. \& Wagenmakers, A. (2000). Relationship between gastro-intestinal complaints and endotoxaemia, cytokine release and the acutephase reaction during and after a long-distance triathlon in highly trained men. Clinical Science, 98, 47-55.

Sherman, M \& Lamb, D. (1988). Nutrition and prolonged exercise. En Lamb, D. \& Murray, R. (Eds.), Perspectives in Exercise Science and Sports Medicine: Prolonged exercise (pp. 224228, 264-276). Dallas, TX: Cooper Publishing Group.
Ulate, E. \& Chinnock, A. (2002). Programa de análisis dietético. San José, Costa Rica: Escuela de Nutrición, Universidad de Costa Rica.

Villa, J., Córdova, A., González, J., Garrido, G. \& Villegas, J. (2000). Nutrición del deportista. España: Gymnos.

Widrick, J., Costill, D., Fink, W., Hickey, M., McConell, G. \& Tanaka, H. (1993). Carbohydrate feedings and exercise performance: effect of initial muscle glycogen concentrations. Journal of Applied Physiology, 74(6), 2998-3005.

Williams, M. (1995). Nutrition for fitness and sport. USA: Brown \& Benchmark Publishers.

Worme, J., Doubt, T., Singh, A., Ryan, C., Moses, F. \& Deuster, P. (1990). Dietary patterns, gastrointestinal complaints, and nutrition knowledge of recreational triathletes. American Journal of Clinical Nutrition, 51(4), 690-697. 